\title{
Effect of high pressure on the crystal structure, magnetic, and vibrational properties of multiferroic $\mathrm{RbFe}\left(\mathrm{MoO}_{4}\right)_{2}$
}

\author{
D. P. Kozlenko, ${ }^{1}$ S. E. Kichanov, ${ }^{1}$ E. V. Lukin, ${ }^{1}$ N. T. Dang, ${ }^{1,2}$ L. S. Dubrovinsky, ${ }^{3}$ E. A. Bykova, ${ }^{3}$ K. V. Kamenev, ${ }^{4}$ \\ H.-P. Liermann, ${ }^{5}$ W. Morgenroth, ${ }^{6}$ A. Ya. Shapiro, ${ }^{7}$ and B. N. Savenko ${ }^{1}$ \\ ${ }^{1}$ Frank Laboratory of Neutron Physics, Joint Institute for Nuclear Research, 141980 Dubna, Russia \\ ${ }^{2}$ Tula State University, Department of Natural Sciences, 300600 Tula, Russia \\ ${ }^{3}$ Bayerisches Geoinstitut, University Bayreuth, D-95440 Bayreuth, Germany \\ ${ }^{4}$ CSEC and School of Engineering, The University of Edinburgh, Edinburgh EH9 3JZ, United Kingdom \\ ${ }^{5}$ Deutsches Elektronen Synchrotron, D-22607 Hamburg, Germany \\ ${ }^{6}$ Institute of Geosciences, University of Frankfurt, D-60438 Frankfurt, Germany \\ ${ }^{7}$ A. V. Shubnikov Institute for Crystallography, RAS, 117333 Moscow, Russia
}

(Received 19 November 2012; revised manuscript received 28 December 2012; published 22 January 2013)

\begin{abstract}
The structural, magnetic, and vibrational properties of $\mathrm{RbFe}\left(\mathrm{MoO}_{4}\right)_{2}$ have been studied by means of $\mathrm{x}$-ray and neutron powder diffraction, magnetic susceptibility measurements, and Raman spectroscopy at pressures up to $10 \mathrm{GPa}$. The gradual structural phase transition from the initial trigonal $P \overline{3} \mathrm{~m} 1$ phase to the monoclinic $C 2 / c$ phase via the intermediate $P \overline{3}$ phase was observed at $P \sim 1 \mathrm{GPa}$. The triangular antiferromagnetic order with a propagation vector $q=\left(1 / 3,1 / 3, q_{\mathrm{z}}\right)$ in the suppressed trigonal phase remains incommensurate, and the $q_{z}$ value increases from 0.45 to 0.48 in the pressure range $0-2 \mathrm{GPa}$ at $T=2 \mathrm{~K}$. The pressure coefficient of the Néel temperature is equal to $\left(1 / T_{N}\right) d T_{N} / d P=0.09 \mathrm{GPa}^{-1}$. No evidence of the formation of the long-range magnetic order in the monoclinic phase was found. The pressure dependencies of the vibrational modes for the trigonal and monoclinic phases were obtained.
\end{abstract}

DOI: 10.1103/PhysRevB.87.014112

PACS number(s): $61.50 . \mathrm{Ks}, 75.25 .-\mathrm{j}, 78.30 .-\mathrm{j}$

\section{INTRODUCTION}

The multiferroic materials, demonstrating a coupling between magnetic and electric subsystems, have become a subject of extensive scientific research in recent years. A coexistence of spontaneous ferroelectric polarization with long-range magnetic order in multiferroics provides a route for the construction of novel electronic devices with a possibility to control magnetic properties with the electric field and vice versa. $^{1-3}$

While in "classical" multiferroics, such as $\mathrm{BiFeO}_{3}$ and hexagonal $R \mathrm{MnO}_{3}(R=\mathrm{Ho}, \mathrm{Y}, \mathrm{Lu}, \ldots)$, the ferroelectric transition temperatures substantially exceed the magnetic ordering temperature, recently, a novel class of materials, characterized by simultaneous occurrence of ferroelectric and magnetic transitions, was discovered. ${ }^{3,4}$ In these multiferroics, inversion symmetry of the crystal structure is broken by incommensurate magnetic arrangement of magnetic moments, allowing for ferroelectricity. An interesting example of such materials, combining multiferroic phenomena with geometrically frustrated magnetism on a two-dimensional triangular lattice, is rubidium molybdate $\left[\mathrm{RbFe}\left(\mathrm{MoO}_{4}\right)_{2}\right]$.

The crystal structure of $\mathrm{RbFe}\left(\mathrm{MoO}_{4}\right)_{2}$ has trigonal $P \overline{3} m 1$ symmetry at ambient conditions, and it is lowered to $P \overline{3}$ via temperature-driven structural distortion below $T=180 \mathrm{~K} .{ }^{6,7}$ The $\mathrm{Fe}^{3+}$ ions with $S=5 / 2$ form regular triangular magnetic planes, separated from each other by layers formed by $\mathrm{MoO}_{4}$ and $\mathrm{Rb}$ ions. The $\mathrm{Fe}^{3+}$ spins in magnetic planes are coupled by superexchange antiferromagnetic interactions via two oxygen anions, while interplane coupling is at least 25 times weaker. The triangular antiferromagnetic order with helical modulation along the $c$ axis and propagation vector $q=(1 / 3$, $\left.1 / 3, q_{z}\right), q_{z} \sim 0.45 \mathrm{rlu}$ (reciprocal lattice units), is formed below $T_{N}=3.8 \mathrm{~K}^{5,8}$
The binary molybdates are known to exhibit a structural polymorphism due to chemical substitution and variation of thermodynamic parameters. ${ }^{9}$ Recent energy-dispersive $\mathrm{x}$-ray diffraction experiments revealed a signature of the structural phase transition in $\mathrm{RbFe}\left(\mathrm{MoO}_{4}\right)_{2}$ at $P \sim 5 \mathrm{GPa}$, and a pressure-induced amorphization was assumed to occur at higher pressures. ${ }^{10}$ In contrast, the subsequent Raman spectroscopy study revealed two structural phase transitions in $\mathrm{RbFe}\left(\mathrm{MoO}_{4}\right)_{2}$ at high pressures, from the $P \overline{3} m 1$ phase to the $P \overline{3}$ one at $P=0.2 \mathrm{GPa}$ and to a phase with unknown lower symmetry at $P \sim 0.4-0.7 \mathrm{GPa} .{ }^{11,12}$ The pressure-induced structural phase transitions at $P \sim 1-2$ GPa were also detected by Raman and x-ray diffraction methods in the relevant compounds $\mathrm{KFe}\left(\mathrm{MoO}_{4}\right)_{2}, \mathrm{KSc}\left(\mathrm{MoO}_{4}\right)_{2}$, and $\mathrm{NaAl}\left(\mathrm{MoO}_{4}\right)_{2}{ }^{5-15}$ However, the high-pressure behavior of structural parameters and physical properties of these compounds in the newly formed phases remain unclear.

In order to study in detail the high-pressure effects on the crystal structure, magnetic, and vibrational properties of multiferroic $\mathrm{RbFe}\left(\mathrm{MoO}_{4}\right)_{2}$, we have performed a comprehensive study using such techniques as x-ray and neutron diffraction, magnetic susceptibility, and Raman measurements in the $0-10 \mathrm{GPa}$ pressure range.

\section{EXPERIMENT}

The single crystals of $\mathrm{RbFe}\left(\mathrm{MoO}_{4}\right)_{2}$ were prepared by a flux method from a mixture of $\mathrm{Rb}_{2} \mathrm{CO}_{3}, \mathrm{Fe}_{2} \mathrm{O}_{3}$, and $\mathrm{MoO}_{3}$ in a ratio 2:1:6, as described in detail in Ref. 7. We used the sample in the single-crystal form for magnetic susceptibility measurements and in the powdered form for $\mathrm{x}$-ray diffraction, Raman spectroscopy, and neutron diffraction measurements. 
Angle-dispersive $\mathrm{x}$-ray powder diffraction patterns at high pressures up to $10 \mathrm{GPa}$ and ambient temperature were measured at the Extreme Conditions Beamline P02.2 (ECB) ${ }^{16}$ at the third-generation synchrotron radiation source PETRA III, located at the Deutsches Elektronen Synchrotron (DESY, Hamburg, Germany.) We used a wavelength of $\lambda=0.29118 \AA$ to collect diffraction images on the amorphous silicon flatpanel detector bonded to a ScI sincilator (XRD 1621) from Perkin Elmer and located at a distance of $402.33 \mathrm{~mm}$ from the sample. The two-dimensional XRD images were converted to one-dimensional diffraction patterns using the FIT2D program. ${ }^{17}$ Raman spectra at ambient temperature and pressures up to $9.5 \mathrm{GPa}$ were collected using a LabRam spectrometer (NeHe excitation laser) with a wavelength of $632.8 \mathrm{~nm}, 1800$ grating, a confocal hole of $1100 \mu \mathrm{m}$, and a $50 \times$ objective. The angle-dispersive $\mathrm{x}$-ray diffraction and Raman spectroscopy experiments have been performed with the same four-pin-type diamond-anvil cell. ${ }^{18}$ The sample was loaded into the hole of the $200-\mu \mathrm{m}$ diameter made in the Re gasket indented to $50 \mu \mathrm{m}$. Diamonds with culets of $400 \mu \mathrm{m}$ were used. Neon gas compressed to $1.5 \mathrm{GPa}$ was used as a pressure-transmitting medium. The pressure was determined by the ruby fluorescence technique.

The magnetic susceptibility measurements were performed using a miniature high-pressure cell for a superconducting quantum interference device (SQUID) magnetometer using a dc field of 100 Oe. ${ }^{19}$ A pair of beveled diamonds with culets of $800 \mu \mathrm{m}$ was used as anvils, and a $250-\mu \mathrm{m}$-thick berylliumcopper foil indented to $100 \mu \mathrm{m}$ was used as the gasket. A hole with a $350-\mu \mathrm{m}$ diameter was drilled in the gasket, and the sample was loaded into the gasket hole. Daphne 7373 oil was used as the pressure-transmitting medium.

The magnetic structure of $\mathrm{RbFe}\left(\mathrm{MoO}_{4}\right)_{2}$ in the pressure range $0-2 \mathrm{GPa}$ and the temperature range $2-5 \mathrm{~K}$ was investigated with the G6.1 diffractometer at the Orphée reactor (Laboratoire Léon Brillouin, Gif-sur-Yvette, France) using a setup for high-pressure experiments. ${ }^{20}$ The incident neutron wavelength was $4.74 \AA$. A sample with a volume of about $2 \mathrm{~mm}^{3}$ was loaded into the sapphire-anvil high-pressure cell. ${ }^{21}$ Several tiny ruby chips were placed at different points of the sample surface, and the pressure was determined by a standard ruby fluorescence technique. Measurements of the pressure distribution on the sample yielded typical pressure inhomogeneities of $\pm 10 \%$. Data collection took on average 5-7 h per pressure/temperature point. The diffraction data for both the crystal and magnetic structure were analyzed by the Rietveld method using the FULLPROF program. ${ }^{22}$

\section{RESULTS AND DISCUSSION}

\section{A. X-ray Diffraction}

$\mathrm{X}$-ray diffraction patterns (XRD) of $\mathrm{RbFe}\left(\mathrm{MoO}_{4}\right)_{2}$ at selected pressures and ambient temperature are shown in Fig. 1. The obtained values of lattice parameters at ambient conditions for the $P \overline{3} m 1$ phase, $a=5.671(3)$ and $c=7.494(5) \AA$, are consistent with previous studies. ${ }^{6,7}$ At moderate pressures $P<1 \mathrm{GPa}$, no changes in diffraction patterns were detected, which could be associated with the $P \overline{3} m 1-P \overline{3}$ structural phase transition, observed in the Raman

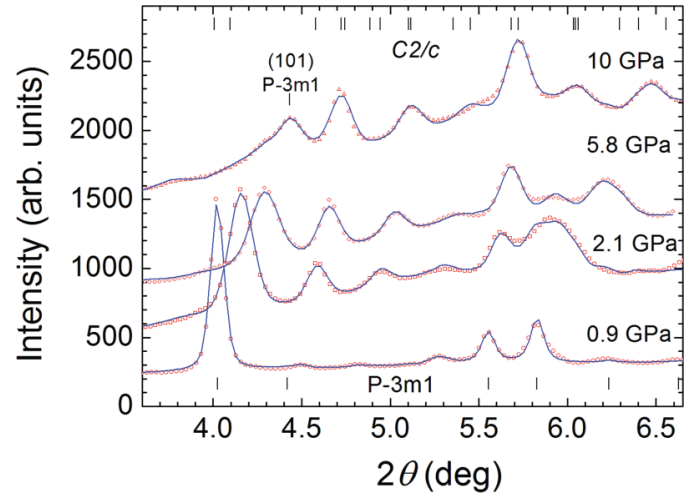

FIG. 1. (Color online) X-ray diffraction patterns of $\mathrm{RbFe}\left(\mathrm{MoO}_{4}\right)_{2}$ measured at selected pressures and ambient temperature and refined by the Rietveld method using FULLPROF. Experimental points and calculated profiles are shown. Tick marks at the bottom and top represent the calculated positions of diffraction peaks for the trigonal phase (at $P=0.9 \mathrm{GPa}$ ) and monoclinic phase (at $P=10 \mathrm{GPa}$ ), respectively.

spectroscopy study at $P=0.2 \mathrm{GPa} .{ }^{11}$ One should note that both phases have very close atomic arrangements, which are difficult to distinguish on the basis of the x-ray data only. ${ }^{7,10} \mathrm{At}$ higher pressures, new diffraction peaks appear at $2 \theta=4.60^{\circ}$, $4.95^{\circ}, 5.73^{\circ}$, and $5.90^{\circ}$, evidencing the structural phase transition. Taking into account possible structural models ${ }^{9}$ of binary molybdates and tungstates of mono- and trivalent elements, successful indexing of diffraction patterns was obtained for the monoclinic unit cell with a $C 2 / c$ symmetry. Lattice parameters are related to those of the initial trigonal cell as $a_{\mathrm{m}} \sim b_{\mathrm{m}} \sim 2 a$, $c_{\mathrm{m}} \sim c$, and $\beta \sim 115^{\circ}$. This model was further used in the Rietveld analysis of the experimental data. During the pressure increase, a gradual suppression of the initial trigonal phase and a stabilization of the high-pressure monoclinic phase was observed. At $P=10 \mathrm{GPa}$, the minor fraction of the trigonal phase was still present in the patterns (Fig. 1). The somewhat larger transition pressure to the monoclinic phase $P \sim 1 \mathrm{GPa}$ and broader phase coexistence range observed in our experiments with powdered sample compared to previous Raman experiments with single crystals ${ }^{11}$ should be related to the presence of pressure gradients due to nonhydrostatic pressure conditions. No traces of amorphization in $\mathrm{RbFe}\left(\mathrm{MoO}_{4}\right)_{2}$ were found upon compression, in contrast to a previous study. ${ }^{10}$ This discrepancy can be explained by the use of the energydispersive $\mathrm{x}$-ray diffraction technique, providing distortion of the intensities of diffraction peaks due to nonlinear $x$-ray absorption, and also noticeably smaller intensities of diffraction peaks of the low-symmetry monoclinic phase in comparison with those of the trigonal phase, as one can see in Fig. 1.

In the $C 2 / c$ monoclinic crystal structure, the $\mathrm{Rb}$ and $\mathrm{Fe}$ atoms occupy positions $4 e\left(0, y, \frac{1}{4}\right)$, and one Mo and four inequivalent $\mathrm{O}$ atoms occupy positions $8 f(x, y, z)$. As starting values, the relevant coordinates of atoms for the similar compound $\mathrm{RbNd}\left(\mathrm{WO}_{4}\right)_{2}$ were used, which crystallizes in $C 2 / c$ structure at ambient conditions and has values of lattice parameters close to those evaluated for the high-pressure phase of $\mathrm{RbFe}\left(\mathrm{MoO}_{4}\right)_{2} \cdot{ }^{23}$ Due to a restricted number of well-separated peaks of the monoclinic phase of $\mathrm{RbFe}\left(\mathrm{MoO}_{4}\right)_{2}$ 


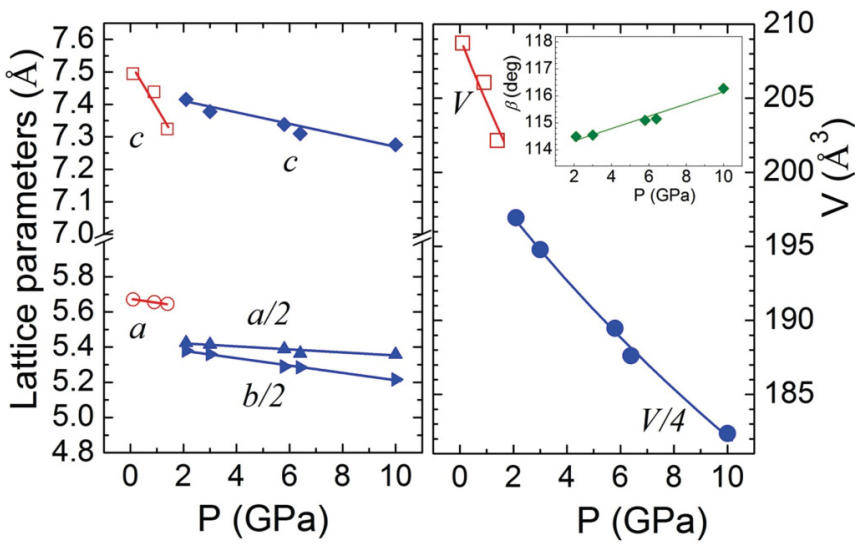

FIG. 2. (Color online) (left) Lattice parameters, (right, inset) monoclinic angle, and (right) unit cell volume as functions of pressure in the trigonal and monoclinic phases of $\mathrm{RbFe}\left(\mathrm{MoO}_{4}\right)_{2}$.

we were able to evaluate the atomic coordinates of $\mathrm{Rb}, \mathrm{Fe}$, and Mo atoms only, while oxygen coordinates exhibited strong correlation in refinements and thus were fixed to those of $\mathrm{RbNd}\left(\mathrm{WO}_{4}\right)_{2}$. The characteristic values of these coordinates at $P=5.8 \mathrm{GPa}$ are $y=0.673(8)$ for $\mathrm{Rb} ; y=0.713(7)$ for $\mathrm{Fe}$; and $x=0.805(7), y=0.502(8), z=0.832(7)$ for Mo atoms; the $R$ factors obtained in Rietveld refinement are $R_{\mathrm{p}}=9.55 \%$ and $R_{\mathrm{wp}}=8.37 \%$. The similarity between monoclinic crystal structures of $\mathrm{RbNd}\left(\mathrm{WO}_{4}\right)_{2}$ and $\mathrm{RbFe}\left(\mathrm{MoO}_{4}\right)_{2}$ implies that in the high-pressure phase of $\mathrm{RbFe}\left(\mathrm{MoO}_{4}\right)_{2}$ the coordination polyhedrons formed by $\mathrm{O}$ atoms around Mo change from tetrahedral to octahedral, and the coordination of polyhedrons formed by $\mathrm{O}$ atoms around $\mathrm{Fe}$ changes from octahedral to dodecahedral.

The pressure dependencies of lattice parameters and unit cell volume of the trigonal and monoclinic phases of $\mathrm{RbFe}\left(\mathrm{MoO}_{4}\right)_{2}$ are shown in Fig. 2. Due to the phase coexistence after the phase transition, their values for the trigonal phase were determined with sufficient accuracy for the pressures up to $2.1 \mathrm{GPa}$ only. At the trigonal-monoclinic phase transition a reduction of the volume per formula unit by about $2.6 \%$ occurs. The lattice compression is anisotropic, with the $c$ axis in the trigonal phase and the $b$ axis in the monoclinic phase most compressible. The monoclinic angle increases upon compression (Fig. 2). The volume compressibility data (Fig. 2) were fitted by the third-order Birch-Murnaghan equation of state: ${ }^{24}$

$$
P=\frac{3}{2} B_{0}\left(x^{-7 / 3}-x^{-5 / 3}\right)\left[1+\frac{3}{4}\left(B^{\prime}-4\right)\left(x^{-2 / 3}-1\right)\right],
$$

where $x=V / V_{0}$ is the relative volume change, $V_{0}$ is the unit cell volume at $P=0$, and $B_{0}$ and $B^{\prime}$ are the bulk modulus $\left[B_{0}=-V(d P / d V)_{\mathrm{T}}\right]$ and its pressure derivative $\left[B^{\prime}=\left(d B_{0} / d P\right)_{T}\right]$. The fitted values $B_{0}=45(5) \mathrm{GPa}$, $B^{\prime}=4.0(5), V_{0}=209.1(4) \AA^{3}$ for the trigonal phase and $B_{0}=80(5) \mathrm{GPa}, B^{\prime}=4.0(5), V_{0}=806.8(6) \AA^{3}$ for the monoclinic phase were obtained.

In the trigonal phase of $\mathrm{RbFe}\left(\mathrm{MoO}_{4}\right)_{2}$, the $\mathrm{Fe}$ atoms form regular triangular (001) planes with an intraplanar $\mathrm{Fe}-\mathrm{Fe}$ distance of $5.67 \AA$ and an interplanar distance of $7.49 \AA$ at ambient pressure. In the monoclinic phase, the pronounced distortion of Fe triangular $(-222)$ planes occurs, with three

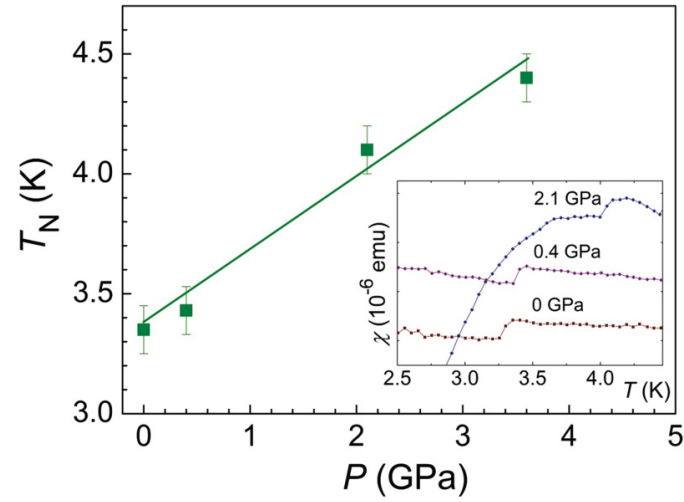

FIG. 3. (Color online) The pressure dependence of the Néel temperature and temperature dependencies of the magnetic susceptibility (inset) of $\mathrm{RbFe}\left(\mathrm{MoO}_{4}\right)_{2}$ at high pressures. The line is a guide for the eye only.

inequivalent $\mathrm{Fe}-\mathrm{Fe}$ intraplanar distances of 5.07, 6.37, and $7.55 \AA$ and an interplanar distance of $7.34 \AA$, as calculated for $P=5.8 \mathrm{GPa}$. As a result, the average nearest-neighbor $\mathrm{Fe}-\mathrm{Fe}$ intraplanar distance is equal to $6.33 \AA$, and it increases by $11 \%$ in comparison with the trigonal phase. This implies a weakening of the magnetic superexchange interactions for the monoclinic phase.

\section{B. Magnetic properties}

The temperature dependencies of the magnetic susceptibility of $\mathrm{RbFe}\left(\mathrm{MoO}_{4}\right)_{2}$, measured at high pressures up to 3.6 GPa, are shown in Fig. 3. They exhibit a characteristic peak corresponding to the onset of the magnetic order in the trigonal $P \overline{3}$ phase. The Néel temperature for this phase increases upon compression, with an average pressure coefficient $\left(1 / T_{N}\right) d T_{N} / d P=0.09 \mathrm{GPa}^{-1}$ (Fig. 3). This value is about half of the value of $0.016 \mathrm{GPa}^{-1}$ found for the hexagonal triangular magnet $\mathrm{YMnO}_{3}$, where $S=2$ spins are coupled by antiferromagnetic (AFM) superexchange interactions via single oxygen anions. ${ }^{25}$ No additional features which could be associated with the formation of magnetic order in the monoclinic phase were observed in the studied pressure range.

In order to study evolution of the magnetic order, additional neutron diffraction experiments were performed (Fig. 4). Due to pronounced pressure gradients related to structural phase coexistence, it was possible to measure data of satisfactory quality at pressures up to $2 \mathrm{GPa}$ only. At ambient pressure and temperatures below $T_{N}=3.8 \mathrm{~K}$, the appearance of the magnetic peaks $\left(-1 / 3,2 / 3,-q_{\mathrm{z}}\right)$ and $\left(2 / 3,-1 / 3,-q_{\mathrm{z}}\right), q_{\mathrm{z}}=$ $0.452(5) \mathrm{rlu}$, was observed, corresponding to the onset of the incommensurate triangular AFM order with a propagation vector $q=\left(1 / 3,1 / 3, q_{z}\right)$ in the trigonal $P \overline{3}$ phase. The obtained value of the ordered magnetic moments of Fe ions is 4.1(1) $\mu_{B}$ at $T=2 \mathrm{~K}$, which is consistent with previous studies. ${ }^{5,8}$ An increase of the $q_{\mathrm{z}}$ value up to $0.480(9) \mathrm{rlu}$ in the pressure range 0-2 GPa was observed [Fig. 4(b)]. It is accompanied by the reduction of the ordered magnetic moment of Fe ions to 3.9(1) $\mu_{B}$ due to gradual suppression of the trigonal phase. The temperature dependencies of the ordered magnetic moments obtained at $P=0$ and $1 \mathrm{GPa}$ [Fig. 4(c)] were interpolated by functions $\mu / \mu_{0} \sim\left[1-\left(T / T_{N}\right)^{\alpha}\right]^{\beta}$, where $\mu_{0}$ is the moment 


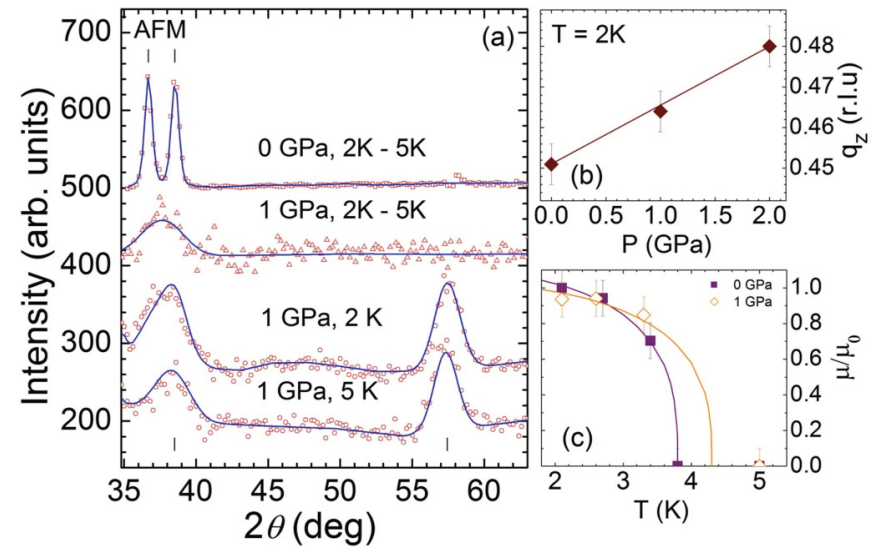

FIG. 4. (Color online) (a) Neutron diffraction patterns of $\mathrm{RbFe}\left(\mathrm{MoO}_{4}\right)_{2}$, measured at $P=1 \mathrm{GPa}, T=2$ and $5 \mathrm{~K}$ and their difference, illustrating magnetic contribution, processed by the Rietveld method. For comparison the difference pattern at $P=0 \mathrm{GPa}$ is also shown. The ticks represent calculated positions of nuclear structure peaks (bottom) and magnetic structure peaks AFM (top). (b) Pressure dependence of the $q_{\mathrm{z}}$ component of the propagation vector, determined at $T=2 \mathrm{~K}$. (c) Temperature dependencies of the ordered Fe magnetic moment at $P=0$ and $1 \mathrm{GPa}$, normalized to the value obtained at $P=0 \mathrm{GPa}$ and $T=2 \mathrm{~K}$. The solid lines represent interpolations as described in the text.

value at $T=0$ and $T_{N}$ is the Néel temperature. The parameter values $\alpha=2.5(1)$ and $\beta=0.32(5)$ were obtained for both pressures. The calculated value of the pressure coefficient of the Néel temperature $\left(1 / T_{N}\right) d T_{N} / d P=0.13 \mathrm{GPa}^{-1}$ is comparable with one obtained from the magnetic susceptibility data. No evidence of the additional magnetic peaks which could be associated with the formation of the magnetic order in the pressure-induced monoclinic phase was observed.

\section{Raman sectroscopy}

The Raman spectra of $\mathrm{RbFe}\left(\mathrm{MoO}_{4}\right)_{2}$ measured at selected pressures and ambient temperature are shown in Fig. 5. At ambient pressure, five lines at 980.4, 933.9, 769.1, 366.3, and

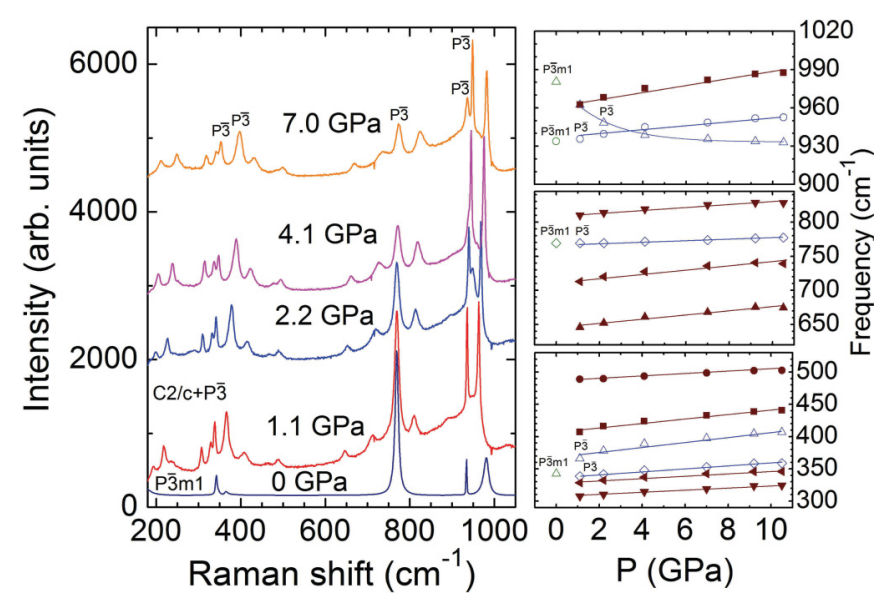

FIG. 5. (Color online) (left) Raman spectra of $\mathrm{RbFe}\left(\mathrm{MoO}_{4}\right)_{2}$ at selected pressures. (right) Pressure dependencies of stretching and bending modes. Open symbols correspond to $P \overline{3} m 1$ and $P \overline{3}$ trigonal phases; solid symbols correspond to the monoclinic phase.
$342.5 \mathrm{~cm}^{-1}$ are detected. For the trigonal crystal structure of $P \overline{3} m 1$ symmetry, group theory predicts nine active Raman modes, $\Gamma_{\text {Ram }}=4 A_{1 g}+5 E_{g} .{ }^{7}$ According to previous studies of $\mathrm{RbFe}\left(\mathrm{MoO}_{4}\right)_{2}$ and related compounds, ${ }^{7,11-14}$ the observed Raman lines correspond to $A_{1 g}\left(980.4,933.9 \mathrm{~cm}^{-1}\right)$ and $E_{g}$ $\left(769.1 \mathrm{~cm}^{-1}\right)$ stretching modes and $E_{g}\left(366.3 \mathrm{~cm}^{-1}\right)$ and $A_{1 g}$ $\left(342.5 \mathrm{~cm}^{-1}\right)$ bending modes of $\mathrm{MoO}_{4}$ tetrahedra. The very narrow width of the $A_{1 g}$ mode at $933.9 \mathrm{~cm}^{-1}$ reflects a weak anharmonicity of the stretching motions of Mo-O1 bonds. ${ }^{12}$

In the spectra measured at $P \sim 1 \mathrm{GPa}$ and higher pressures, two effects were found. First, an appearance of a number of additional Raman modes was observed (Fig. 5), indicating the structural phase transition to the lower symmetry monoclinic phase. In addition, a strong increase of the intensity of the bending mode peak at $366 \mathrm{~cm}^{-1}$ is detected, which is a characteristic feature of the structural transition between the $P \overline{3} m 1$ and $P \overline{3}$ phases, expected to occur at $P=0.2 \mathrm{GPa} .{ }^{11}$ The intensities of newly appearing Raman peaks increased under pressure, while those corresponding to the trigonal phase decreased (Fig. 5). This behavior reflects the phase coexistence of the gradually suppressed trigonal phase in favor of the newly formed monoclinic high-pressure phase, in accordance with x-ray diffraction measurements. In the trigonal $P \overline{3}$ phase the frequencies of most of the observed modes increase nearly linearly upon compression, and their pressure coefficients $\left(1 / v_{i 0}\right) d v_{i} / d P$ are given in Table I. The $A_{1 g}$ stretching mode exhibits anomalous nonlinear softening under pressure, and its average pressure coefficient changes from $-0.008 \mathrm{GPa}^{-1}$ in the 1.1-4.1 $\mathrm{GPa}$ pressure range to $-0.001 \mathrm{GPa}^{-1}$ at higher pressures. A pronounced softening of this mode was also previously observed in the Raman study of single crystals of $\mathrm{RbFe}(\mathrm{MoO} 4)_{2}$, and it is related to the enlargement of the Mo-O1 bonds under pressure, as shown by density functional theory calculations. ${ }^{11}$

The contribution to Raman spectra from the high-pressure phase of $\mathrm{RbFe}\left(\mathrm{MoO}_{4}\right)_{2}$ formed above $1 \mathrm{GPa}$ resembles that of the $\mathrm{RbNd}\left(\mathrm{WO}_{4}\right)_{2}$ compound, having the monoclinic $C 2 / c$ symmetry at ambient conditions. ${ }^{26}$ This provides additional support to the assignment of the $C 2 / c$ monoclinic group for the high-pressure phase of $\mathrm{RbFe}\left(\mathrm{MoO}_{4}\right)_{2}$. For the monoclinic crystal structure of the $C 2 / c$ symmetry, the group theory predicts 36 Raman active modes, $\Gamma_{\text {Ram }}=17 A_{g}+19 B_{g}$. The assignment of the Raman lines for the high-pressure monoclinic phase of $\mathrm{RbFe}\left(\mathrm{MoO}_{4}\right)_{2}$, based on experimental and theoretical results, ${ }^{11,26}$ is presented in the Table I along with the corresponding pressure coefficients. The modes located in the region $975-660 \mathrm{~cm}^{-1}$ involve the stretching vibrations of the Mo-O1, 2, 3, 4 bonds. This region is much broader in comparison with the trigonal phase, implying the enhanced difference in the values of inequivalent Mo-O bonds. The modes located in the region of $495-314 \mathrm{~cm}^{-1}$ involve the bending vibrations of the double oxygen bridges Mo-O-O-Mo, single oxygen bridges Mo-O-Mo, and Mo-O3, 4 bonds. The modes located below $250 \mathrm{~cm}^{-1}$ correspond to translational vibrations of $\mathrm{Mo}, \mathrm{Fe}$, and $\mathrm{Nd}$ atoms. ${ }^{26}$

The pressure coefficients of the stretching modes are positive and they have rather different values from 0.002 to $0.005 \mathrm{GPa}^{-1}$, reflecting the anisotropic deformation of inequivalent Mo-O bonds. These values are comparable with those found in the Raman study of $\mathrm{RbFe}\left(\mathrm{MoO}_{4}\right)_{2}$ 
TABLE I. Assignment and pressure coefficients $\left[(1 / v i 0) d v_{i} / d P\right]$ of Raman modes in the trigonal $P \overline{3}$ and monoclinic $C 2 / c$ phases of $\mathrm{RbFe}\left(\mathrm{MoO}_{4}\right)_{2}$. The frequency values obtained at $P=4.1 \mathrm{GPa}$ are given.

\begin{tabular}{|c|c|c|c|c|c|}
\hline \multicolumn{3}{|c|}{ Trigonal phase $(P \overline{3})$} & \multicolumn{3}{|c|}{ Monoclinic phase $(C 2 / c)$} \\
\hline $\begin{array}{l}\text { Mode } \\
\left(\mathrm{cm}^{-1}\right)\end{array}$ & Assignment & $\begin{array}{c}\text { Pressure } \\
\text { coefficient }\left(\mathrm{GPa}^{-1}\right)\end{array}$ & $\begin{array}{l}\text { Mode } \\
\left(\mathrm{cm}^{-1}\right)\end{array}$ & Assignment & $\begin{array}{c}\text { Pressure } \\
\text { coefficient }\left(\mathrm{GPa}^{-1}\right)\end{array}$ \\
\hline 938.7 & $A_{1 g}$, stretching $\left(\mathrm{MoO}_{4}\right)$ & $\begin{array}{c}-0.008 \\
(P \leqslant 4.1 \mathrm{GPa})\end{array}$ & 975.3 & $\begin{array}{c}A_{g}, B_{g}, \text { stretching } \\
\text { (Mo-O bonds) }\end{array}$ & 0.003 \\
\hline 944.9 & $A_{1 g}$, stretching $\left(\mathrm{MoO}_{4}\right)$ & 0.002 & 818.8 & & 0.002 \\
\hline 771.2 & $E_{g}$, stretching $\left(\mathrm{MoO}_{4}\right)$ & 0.001 & 727.5 & & 0.004 \\
\hline 388.9 & $E_{g}$, bending $\left(\mathrm{MoO}_{4}\right)$ & 0.012 & 660.9 & & 0.005 \\
\hline \multirow[t]{6}{*}{347.7} & $E_{g}$, bending $\left(\mathrm{MoO}_{4}\right)$ & 0.006 & 493.8 & $A_{g}, B_{g}$, bending & 0.003 \\
\hline & & & 423.7 & (Mo-O-Mo and & 0.009 \\
\hline & & & 336.9 & Mo-O-O-Mo & 0.006 \\
\hline & & & 314.6 & $\begin{array}{l}\text { bridges, Mo-O } \\
\text { bonds) }\end{array}$ & 0.006 \\
\hline & & & 238.8 & Translation & 0.020 \\
\hline & & & 205.0 & (Mo, $\mathrm{Rb}$, and $\mathrm{Fe}$ ) & 0.013 \\
\hline
\end{tabular}

single crystals in more restricted pressure range. ${ }^{11}$ A similar pressure behavior of the stretching modes was also observed in the previous studies of $\mathrm{RbFe}\left(\mathrm{MoO}_{4}\right)_{2}, \mathrm{RbNd}\left(\mathrm{WO}_{4}\right)_{2}$ and $\mathrm{NaAl}\left(\mathrm{MoO}_{4}\right)_{2} \cdot{ }^{11,15,26}$

The pressure coefficients of the observed bending and translational modes are positive. They reach values of 0.01$0.02 \mathrm{GPa}^{-1}$ for translational modes (Table I), much larger compared with ones for the stretching modes. This assumes the largest compressibility of the $\mathrm{Fe}-\mathrm{O}$ bonds in comparison with the Mo-O and $\mathrm{Rb}-\mathrm{O}$ bonds.

\section{CONCLUSIONS}

Our results demonstrate that the application of high pressure $P>1$ GPa leads to the formation of the monoclinic phase of $C 2 / c$ symmetry in $\mathrm{RbFe}\left(\mathrm{MoO}_{4}\right)_{2}$. This indicates a tendency towards stabilization of structures with a denser atomic packing and an increased coordination number of oxygen polyhedrons around monovalent and trivalent elements in binary molybdates and tungstates upon compression.

In the trigonal $P \overline{3}$ phase the $q_{z}$ component of the propagation vector, corresponding to the triangular antiferromagnetic magnetic order, approaches the commensurate value of $1 / 2$ upon compression. The pressure coefficient of the Néel temperature for $\mathrm{RbFe}\left(\mathrm{MoO}_{4}\right)_{2}$ with superexchange interactions via two anions is significantly lower compared with relevant systems possessing superexchange interactions via one anion. No sign of a long-range magnetic order formation in the monoclinic phase was observed in the studied pressure and temperature range. This is consistent with the observed distortion of the triangular lattice formed by $\mathrm{Fe}^{3+}$ ions, giving rise to an increase in the average Fe-Fe distance. The Raman spectroscopy data demonstrate the anisotropic deformation of $\mathrm{Fe}-\mathrm{O}$ and $\mathrm{Mo}-\mathrm{O}$ polyhedrons with the most compressible $\mathrm{Fe}-\mathrm{O}$ bonds compared with the Mo-O and $\mathrm{Rb}-\mathrm{O}$ ones in the monoclinic phase.

\section{ACKNOWLEDGMENTS}

This work has been supported by Grants RFBR No. 12-0200794-a and No. MD-3125.2013.2. The authors acknowledge the useful discussions and technical assistance of A. I. Smirnov, I. Mirebeau, and O. L. Makarova, as well as financial support from Laboratoire Léon Brillouin. W.M. also gratefully acknowledges the financial support from BMBF (Project No. 05K10RFA).

\footnotetext{
${ }^{1}$ M. Fiebig, J. Phys. D 38, R123 (2005).

${ }^{2}$ T. Kimura, T. Goto, H. Shintani, K. Ishizaka, T. Arima, and

Y. Tokura, Nature (London) 426, 55 (2003).

${ }^{3}$ S.-W. Cheong and M. Mostovoy, Nat. Mater. 6, 13 (2007).

${ }^{4}$ G. A. Smolenskii and I. E. Chupis, Sov. Phys. Usp. 25, 475 (1982).

${ }^{5}$ M. Kenzelmann, G. Lawes, A. B. Harris, G. Gasparovic,

C. Broholm, A. P. Ramirez, G. A. Jorge, M. Jaime, S. Park,

Q. Huang, A.Ya. Shapiro, and L. A. Demianets, Phys. Rev. Lett.

98, 267205 (2007).

${ }^{6}$ R. F. Klevtsova and P. V. Klevtsov, Kristallografiya 15, 953 (1970).
}

${ }^{7}$ S. A. Klimin, M. N. Popova, B. N. Mavrin, P. H. M. van Loosdrecht, L. E. Svistov, A. I. Smirnov, L. A. Prozorova, H.-A. Krug von Nidda, Z. Seidov, A. Loidl, A. Ya. Shapiro, and L. A. Demianets, Phys. Rev. B 68, 174408 (2003).

${ }^{8}$ L. E. Svistov, A. I. Smirnov, L. A. Prozorova, O. A. Petrenko, L. A. Demianets, and A. Ya. Shapiro, Phys. Rev. B 67, 094434 (2003).

${ }^{9}$ V. A. Isupov, Ferroelectrics 321, 63 (2005).

${ }^{10}$ A. Waskowska, L. Gerward, J. Staun Olsen, W. Morgenroth, M. Maczka, and K. Hermanowicz, J. Phys. Condens. Matter 22, 055406 (2010). 
${ }^{11}$ M. Maczka, M. Ptak, C. Luz-Lima, P. T. C. Freire, W. Paraguassu, S. Guerini, and J. Hanuza, J. Solid State Chem. 184, 2812 (2011).

${ }^{12}$ M. Maczka, A. G. Souza-Filho, W. Paraguassu, P. T. C. Freire, J. Mendes Filho, and J. Hanuza, Prog. Mater. Sci. 57, 1335 (2012).

${ }^{13}$ M. Maczka, A. Pietraszko, G. D. Saraiva, A. G. Souza Filho, W. Paraguassu, V. Lemos, C. A. Perottoni, M. R. Gallas, P. T. C. Freire, P. E. Tomaszewski, F. E. A. Melo, J. Mendes Filho, and J. Hanuza, J. Phys. Condens. Matter 17, 6285 (2005).

${ }^{14}$ G. D. Saraiva, M. Maczka, P. T. C. Freire, J. Mendes Filho, F. E. A. Melo, J. Hanuza, Y. Morioka, and A. G. Souza Filho, Phys. Rev. B 67, 224108 (2003).

${ }^{15}$ W. Paraguassu, A. G. Souza Filho, M. Maczka, P. T. C. Freire, F. E. A. Melo, J. Mendes Filho, and J. Hanuza, J. Phys. Condens. Matter 16, 5151 (2004).

${ }^{16}$ H.-P. Liermann, W. Morgenroth, A. Ehnes, A. Berhauser, B. Winkler, H. Franz, and E. Weckert, J. Phys. Conf. Ser. 215, 012029 (2010).

${ }^{17}$ A. P. Hammersley, S. O. Svensson, M. Hanfland, A. N. Fitch, and D. Hausermann, High Pressure Res. 14, 235 (1996).
${ }^{18}$ N. A. Dubrovinskaia and L. S. Dubrovinsky, Rev. Sci. Instrum. 74, 3433 (2003).

${ }^{19}$ G. Giriat, W. Wang, J. P. Attfield, A. D. Huxley, K. V. Kamenev, Rev. Sci. Instrum. 81, 073905 (2010).

${ }^{20}$ I. N. Goncharenko, I. Mirebeau, P. Molina, and P. Boni, Phys. B 234-236, 1047 (1997).

${ }^{21}$ I. N. Goncharenko, V. P. Glazkov, A. V. Irodova, O. A. Lavrova, and V. A. Somenkov, J. Alloys Compd. 179, 253 (1992); I. N. Goncharenko, High Pressure Res. 24, 193 (2004).

${ }^{22}$ J. Rodríguez-Carvajal, Phys. B 192, 55 (1993).

${ }^{23}$ M. T. Borowiec, A. D. Prokhorov, I. M. Krygin, V. P. Dyakonov, K. Wozniak, L. Dobrzycki, T. Zayarnyuk, M. Baranski, W. Domuchowski, and H. Szymczak, Phys. B 371, 205 (2006).

${ }^{24}$ F. J. Birch, J. Geophys. Res. 91, 4949 (1986).

${ }^{25}$ D. P. Kozlenko, I. Mirebeau, J.-G. Park, I. N. Goncharenko, S. Lee, Junghwan Park, and B. N. Savenko, Phys. Rev. B 78, 054401 (2008).

${ }^{26}$ W. Paraguassu, M. Maczka, S. Guerini, P. T. C. Freire, J. Mendes Filho, A. Majchrowki, and M. Swirkowicz, J. Phys. Condens. Matter 23, 405901 (2011). 